Pacific Journal of Mathematics

CHARACTERIZATIONS OF (H)PI EXTENSIONS 


\title{
CHARACTERIZATIONS OF (H)PI EXTENSIONS
}

\author{
JAAP VAN DER WOUDE
}

\begin{abstract}
A generalization of $\mathbf{I}$. U. Bronstein's characterization for PD extensions is given and, exploiting similar ideas, HPI extensions are characterized intrinsically.
\end{abstract}

I. Introduction. Among the most important features in topological dynamics in order to classify the minimal flows are the PI and HPI towers for homomorphisms (extensions) of minimal flows ([EGS 75], [AG 77] and [V 77]). The techniques involved depend on transfinite induction, on hyperspaces of transfinite degree and also on rather uncontrollable algebraic features in subgroups of the universal minimal flow.

Although this theory is elegant and powerful, it would be more satisfactory if these techniques and properties were related to some internal structure, only using (finite powers of) the flow itself or the fibered product (powers).

In [B 77] such an intrinsic description was given for PI extensions of metric minimal flows. We shall prove a nonmetric version in $\S 3$, and we relate this characterization to the one given in [MN 80] (the relativized version there of).

In $\S 4$ we shall give a similar kind of characterization for HPI extensions.

I would like to thank Ta-Sun $\mathrm{Wu}$ for the many stimulating and encouraging conversations.

In the following we establish some notations, definitions and facts; for more detailed discussions the reader is referred to [G 76] and [Wo 82].

A topological transformation group (ttg or flow) $\mathscr{X}$ is a triple $\mathscr{X}=$ $\langle T, X, \pi\rangle$, where $T$ is a $\mathrm{T}_{2}$ topological group, $X$ is a compact $\mathrm{T}_{2}$ space with unique uniformity $\mathscr{U}_{X}$ and $\pi$ is a jointly continuous action $\pi: T \times X \rightarrow X$. We shall consider ttgs for a fixed (but arbitrary) topological group $T$ and we shall suppress the action symbol, writing the action as a (left) multiplication. A ttg will then be denoted by $\mathscr{X}$ or by its phase space $X$ only. If $x \in X$, then $T x(\overline{T x})$ is the orbit (closure) of $x$ and a subset $A \subseteq X$ is invariant if $T x \subseteq A$ for every $x \in A$, i.e. $T A \subseteq A$. A ttg $\mathscr{X}$ is minimal if $\overline{T x}=X$ for every $x \in X$ or, equivalently, if $X$ does not contain proper closed invariant subsets. If $x \in X$ has a minimal orbit closure, $x$ is called 
an almost periodic point. Note that $\mathscr{X}$ is minimal if for every nonempty open $U \subseteq X$ there is a finite subset $F \subseteq T$ such that $X=F U=T U$. A ttg $\mathscr{X}$ is called ergodic if $X$ does not contain proper closed invariant subsets with nonempty interior or, equivalently, if $X=\overline{T U}$ for every nonempty open $U \subseteq X$.

A homomorphism (extension) of ttgs $\phi: \mathscr{X} \rightarrow \mathscr{Y}$ is a continuous map $\phi:$ $X \rightarrow Y$ that commutes with the action of $T$ (equivariance), i.e. $\phi(t x)=$ $t \phi(x)$ for $t \in T, x \in X$. Unless stated otherwise, a homomorphism of ttgs will be assumed to be a surjection. Let $\phi: \mathscr{X} \rightarrow \mathscr{Y}$ and $\psi: \mathscr{Z} \rightarrow \mathscr{Y}$ be homomorphisms of ttgs. Then define:

$R_{\phi \psi}:=\{(x, z) \in X \times Z \mid \phi(x)=\psi(z)\}$, the fibered product of $\phi$ and $\psi$;

$R_{\phi}:=R_{\phi \phi}=\left\{\left(x_{1}, x_{2}\right) \in X \times X \mid \phi\left(x_{1}\right)=\phi\left(x_{2}\right)\right\}$;

$R_{\phi}^{n}:=\left\{\left(x_{1}, \ldots, x_{n}\right) \in X^{n} \mid \phi\left(x_{1}\right)=\cdots=\phi\left(x_{n}\right)\right\}$,

the fibered $n$-power of $\phi$;

$P_{\phi}:=\bigcap\left\{T \alpha \cap R_{\phi} \mid \alpha \in \mathscr{U}_{X}\right\}$, the relative proximal relation for $\phi$;

$Q_{\phi}:=\bigcap\left\{\overline{T \alpha \cap R_{\phi}} \mid \alpha \in \mathscr{U}_{X}\right\}$, the relative regionally proximal relation for $\phi$ and

$E_{\phi}$, the smallest closed invariant equivalence relation containing

$Q_{\phi}$, the equicontinuous structure relation.

Note that the action of $T$ on powers of $X$ is coordinatewise.

The homomorphism $\phi$ is called almost periodic if $Q_{\phi}=\Delta_{X}$ and proximal if $P_{\phi}=R_{\phi}$. The map $\theta: X / E_{\phi} \rightarrow Y$ is the maximal almost periodic factor of $\phi$, so $\theta$ is nontrivial iff $R_{\phi} \neq E_{\phi}$. A homomorphism $\phi$ : $\mathscr{X} \rightarrow \mathscr{Y}$ of minimal ttgs is called highly proximal $(h p)$ if for some $y \in Y$, the whole fiber $\phi^{\leftarrow}(y)$ of $y$ under $\phi$ shrinks to a point (singleton set) under the action of $T$ on the hyperspace $2^{X}$ of $X$ endowed with the usual Vietoris topology. I.e., for some (any) $y \in Y$ there is a net $\left\{t_{i}\right\}_{i}$ in $T$ such that $t_{i} \phi^{\leftarrow}(y) \rightarrow\{x\}$ in $2^{X}$. Clearly, a highly proximal extension is proximal. One can show that $\phi$ is hp iff $\phi$ is an irreducible map (if $A=\bar{A}$ and $\phi[A]=Y$ then $A=X$ ), e.g. see [AG 77].

Proximal, highly proximal and almost periodic extensions are considered to be basic building blocks in determining the structure of minimal ttgs. For instance in [V 77] it was shown that every extension is built up by proximal and almost periodic extensions up to some weakly mixing "junk". 
A homomorphism $\phi: \mathscr{X} \rightarrow \mathscr{Y}$ is a strictly PI extension if there is an ordinal $\nu$ and a collection $\left\{\phi_{\alpha}^{\beta}: \mathscr{X}_{\beta} \rightarrow \mathscr{X}_{\alpha} \mid \alpha \leq \beta \leq \nu\right\}$ of homomorphisms of minimal ttgs such that

(a) $\mathscr{X}_{0}=\mathscr{Y} ; \mathscr{X}_{\nu}=\mathscr{X} ; \phi_{0}^{\nu}=\phi$;

(b) $\phi_{\alpha}^{\gamma} \circ \phi_{\gamma}^{\beta}=\phi_{\alpha}^{\beta}$ for $\alpha \leq \gamma \leq \beta \leq \nu$;

(c) if $\beta$ is $\operatorname{limit}$ ordinal then $\phi_{\alpha}^{\beta}=\operatorname{inv} \lim \left\{\phi_{\alpha}^{\gamma} \mid \alpha \leq \gamma<\beta\right\}$;

(d) $\phi_{\alpha}^{\alpha+1}$ is either proximal or almost periodic for every $\alpha<\nu$. The collection $\left\{\phi_{\alpha}^{\alpha+1}: \mathscr{X}_{\alpha+1} \rightarrow \mathscr{X}_{\alpha} \mid \alpha<\nu\right\}$ is called the strictly PI tower for $\phi$.

A homomorphism $\phi: \mathscr{X} \rightarrow \mathscr{Y}$ is called a strictly HPI extension if it is a strictly PI extension where every proximal $\phi_{\alpha}^{\alpha+1}$ is highly proximal. The map $\phi$ is called an $(\mathrm{H}) \mathrm{PI}$-extension if there is a strictly $(\mathrm{H}) \mathrm{PI}$-extension $\psi$ : $\mathscr{Z} \rightarrow \mathscr{Y}$ and a (highly) proximal $\theta: \mathscr{Z} \rightarrow \mathscr{X}$ with $\psi=\phi \circ \theta$.

One can show that factors of $(\mathrm{H}) \mathrm{PI}$ extensions are $(\mathrm{H}) \mathrm{PI}$ extensions again; i.e.: if $\phi: \mathscr{X} \rightarrow \mathscr{Y}$ is an (H)PI extension and $\phi=\mu \circ \lambda$ then $\mu$ is an (H)PI extension.

A last remark in this introduction is concerned with some maximal ttgs.

There exists a universal minimal ttg $\mathscr{M}$ for $T$ with phase space $M . M$ may be considered as a minimal left ideal in $S_{T}$, the universal ambit (point transitive flow with distinguished point) for $T$. $M$ inherits a semigroup structure and we write $M=J G$ where $G$ is a maximal subgroup of $M$ and $J$ is the collection of idempotents in $M$. The semigroup $M$ acts on every $\operatorname{ttg} \mathscr{X}$ as the "limit action" of $T$. Note that $x \in X$ is an almost periodic point iff $x=u x$ for some $u \in J$.

If $\mathscr{X}$ is a minimal ttg, then there exists a maximal minimal proximal extension $\mathfrak{A}(\mathscr{X})$ of $\mathscr{X}$; i.e., $\mathfrak{A}(\mathscr{X}) \rightarrow \mathscr{X}$ is proximal and has every proximal extension of $\mathscr{X}$ as a factor. The $\operatorname{tg} \mathfrak{A}(\mathscr{X})$ can be characterized as a certain quasifactor of $\mathscr{M}$; i.e. a minimal sub ttg of the hyper $\operatorname{ttg} 2^{\mathscr{M}}$ (e.g. see [EGS 75] or [G 76]), as follows: let $G=u M$, a maximal subgroup of $M$; here $u \in J$. For $x \in X$ define the Ellis group $H=\mathscr{G}(\mathscr{X}, u x)=\{g \in G \mid g x=$ $u x\}$. Then $\mathfrak{A}(\mathscr{X}):=\mathscr{Q} \mathscr{F}(u \circ H, \mathscr{M})$, mostly denoted by $\mathfrak{U}(H)$, is the quasifactor of $\mathscr{M}$ which is the minimal orbit closure of the closed subset

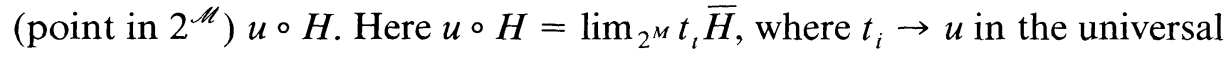
ambit $S_{T}$. I.e., the "circle operation" refers to the action of $M$ on hyperspaces.

If $\phi: \mathscr{X} \rightarrow \mathscr{Y}$ is a homomorphism of minimal ttgs then $\phi$ can be lifted to $\mathfrak{A}(\phi): \mathfrak{U}(\mathscr{X}) \rightarrow \mathfrak{A}(\mathscr{Y})$ which is a RIC extension. RIC extensions will be very important in this paper. An extension $\psi: \mathscr{W} \rightarrow \mathscr{Z}$ of minimal ttgs is 
called a RIC extension if $R_{\psi \theta}$ is minimal for every proximal extension $\theta$ : $\mathscr{W}^{\prime} \rightarrow \mathscr{Z}$. Another, equivalent, definition could be $\psi$ is a RIC extension iff $\psi^{\leftarrow}(z)=u \circ u \psi^{\leftarrow}(z)$ for every $z \in \mathscr{Z}, u \in I_{z}=\{w \in I \mid w z=z\}$.

For a minimal $\operatorname{ttg} \mathscr{X}$ there also exists a maximal highly proximal extension $\mathscr{X}^{*}$ of $\mathscr{X}$ and every homomorphism $\phi: \mathscr{X} \rightarrow \mathscr{Y}$ can be lifted to a map $\phi^{*}: \mathscr{X}^{*} \rightarrow \mathscr{Y}^{*}$ which is an open extension. The $\operatorname{ttg} \mathscr{X}^{*}$ can also be viewed as a quasifactor of $\mathscr{M}$. Moreover, $\mathscr{X}^{*}$ may be characterized as follows: $\mathscr{X}=\mathscr{X}^{*}$ iff every extension which values in $\mathscr{X}$ is open. For more details on $\mathscr{X}^{*}$ and hp extensions see [AG 77] and [AW 81].

For the remainder of this paper it might be useful to remember that $\phi: \mathscr{X} \rightarrow \mathscr{Y}$ is a weakly mixing extension iff $R_{\phi}$ is ergodic and that $\phi$ is said to satisfy the Bronstein condition (Bc) iff $R_{\phi}$ has a dense set of almost periodic points $\left(R_{\phi}=\overline{J R_{\phi}}\right)$. Examples of $\mathrm{Bc}$ extensions are almost periodic extensions and RIC extensions. Examples of weakly mixing exten-

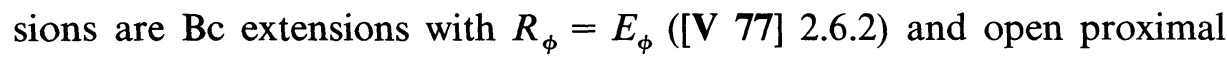
extensions ([Wo 82] VII.2.14).

II. Some basic tools. In this section we gather several useful facts, lemmas and theorems, mainly for the sake of reference and comparison. The cohesive theme is the question about properties of the domain of a homomorphism if the range is ergodic or minimal.

2.1. THEOREM. Let $\phi: \mathscr{X} \rightarrow \mathscr{Y}$ be an almost periodic homomorphism of ttgs with $\mathscr{Y}$ minimal. Then $\mathscr{X}$ is ergodic iff $\mathscr{X}$ is minimal ([AMWW 83] 2.15).

2.2. THEOREM. Let $\phi: \mathscr{X} \rightarrow \mathscr{Y}$ be a proximal homomorphism of ttgs with $\mathscr{Y}$ ergodic. If $X$ has a dense set of almost periodic points, then $\mathscr{X}$ is ergodic ([AMWW 83] 2.10).

2.3. THEOREM. Let $\phi: \mathscr{X} \rightarrow \mathscr{Y}$ be a homomorphism of ttgs such that $\phi:$ $X \rightarrow Y$ is an irreducible map. Then $\mathscr{X}$ is ergodic (minimal) iff $\mathscr{Y}$ is ergodic (minimal).

Proof. The statement about minimality is obvious.

Suppose $\mathscr{Y}$ is ergodic and let $A=\overline{T A} \subseteq X$ have a nonempty interior. As either $\phi[A]$ or $\phi\left[X \backslash A^{\circ}\right]$ has a nonempty interior in $Y$, ergodicity of $\mathscr{Y}$ implies either $Y=\phi[A]$ or $Y=\phi\left[X \backslash A^{\circ}\right]$. By irreducibility of $\varphi, X=A$ or $X=X \backslash A^{\circ}$; but as $A^{\circ} \neq \varnothing$ it follows that $X=A$.

In the fourth section, the notion of semi-openness is very important. Remember that a map $f: X \rightarrow Y$ is semi-open if for every open $U \subseteq X$ the image $f[U]$ has a nonempty interior in $Y$. 
The following remark gives some useful examples of semi-open maps:

2.4. Remark. Let $\phi: \mathscr{X} \rightarrow \mathscr{Y}$ be a homomorphism of ttgs.

(a) If $\phi$ is irreducible then $\phi$ is semi-open; even: for every open $U \subseteq X$ there is an open $U^{\prime} \subseteq U$ with $\overline{U^{\prime}}=\bar{U}$ such that $\phi^{\leftarrow} \phi\left[U^{\prime}\right]=U^{\prime}$, and $\phi\left[U^{\prime}\right]$ is open in $Y$.

(b) If $\mathscr{Y}$ is minimal and if $X$ has a dense set of almost periodic points, then $\phi$ is semi-open. In particular, every homomorphism of minimal ttgs is semi-open.

(c) If $\phi: \mathscr{X} \rightarrow \mathscr{Y}$ is open, then for every $n \in N, n \geq 2$ and for every $m \in \mathbf{N}$ with $m \leq n$ the projection $\pi_{m}: \mathscr{R}_{\phi}^{n} \rightarrow \mathscr{X}$ onto the $m$ th coordinate is semi-open.

The following result deals with the semi-open lifting of ergodicity. It is the "ergodic" counterpart of the fact that the preimage of an almost periodic point contains an almost periodic point.

2.5. TheOREM. Let $\phi: \mathscr{X} \rightarrow \mathscr{Y}$ be a homomorphism of ttgs with $\mathscr{Y}$ ergodic. Then there exists an ergodic subttg $\mathscr{A}$ of $\mathscr{X}$ such that $\left.\phi\right|_{A}: \mathscr{A} \rightarrow \mathscr{Y}$ is a semi-open surjection.

Proof. Let $\mathscr{C}$ be the collection of all closed invariant subsets of $X$ that are mapped onto $Y$ by $\phi$. Then $\mathscr{C}$ is nonempty and ordered by inclusion. It is easily seen that $\mathscr{C}$ is inductively ordered, so by Zorn's lemma there is an $A=\overline{T A} \subseteq X$ with $\phi^{\prime}=\left.\phi\right|_{A}: A \rightarrow Y$ is surjective and that is minimal under that condition. We shall show that $A$ is ergodic and that $\phi^{\prime}$ is semi-open.

Let $F \subseteq A$ be a closed subset of $A$ such that $F$ is mapped irreducibly onto $Y$ by $\left.\phi^{\prime}\right|_{F}$ (e.g.: [Wi 70] 14.2.1). As every $t \in T$ acts as a homeomorphism on $A$, also $\left.\phi^{\prime}\right|_{t F}: t F \rightarrow Y$ is an irreducible surjection for every $t \in T$. Hence, for every $t \in T,\left.\phi^{\prime}\right|_{t F}: t F \rightarrow Y$ is semi-open (compare 2.4(a) where equivariance is not needed).

As $\left.\phi^{\prime}\right|_{\overline{T F}}: \overline{T F} \rightarrow Y$ is a surjection and $\overline{T F} \subseteq A$, it follows that $\overline{T F}=A$. Suppose $U \subseteq A$ is open in $A$. Then for some $t \in T, U \cap t F \neq \varnothing$; so $U \cap t F$ is open in $t F$. By irreducibility of $\left.\phi^{\prime}\right|_{t F}$ we know that $\left.\phi^{\prime}\right|_{t F}[U \cap t F]$ has a nonempty interior in $Y$. Consequently $\left.\phi^{\prime}\right|_{\overline{T F}}[U]$ has a nonempty interior in $Y$, which proves semi-openness for $\left.\phi^{\prime}\right|_{\overline{T F}}=\left.\phi^{\prime}\right|_{A}$. Moreover, ergodicity of $\mathscr{Y}$ implies that $\left.\phi^{\prime}\right|_{A}[\overline{T U}]=\overline{\left.T \cdot \phi^{\prime}\right|_{A}[U]}=Y$. But then by the construction of $A$ it follows that $A=\overline{T U}$. Hence $A$ is ergodic. 
III. A characterization for PI extensions. In [B 77] it was proven that a homomorphism $\phi: \mathscr{X} \rightarrow \mathscr{Y}$ of metric minimal ttgs is a PI extension iff every orbit closure in $R_{\phi}$ with a dense set of almost periodic points is minimal.

Before we state the non-metric version we need the following definition: Let $n \in \mathbf{N}, n \geq 2$; a homomorphism $\phi: \mathscr{X} \rightarrow \mathscr{Y}$ is said to satisfy the $n$ - $\mathrm{C}^{\prime}$-condition (or $\phi$ is an $n$-C'-extension) iff every ergodic subset $A=\overline{T A}$ of $R_{\phi}^{n}$ with a dense set of almost periodic points is minimal. We say that $\phi$ is a $C^{\prime}$-extension if $\phi$ is $2-C^{\prime}$. We shall prove:

3.1. TheOREM. Let $\phi: \mathscr{X} \rightarrow \mathscr{Y}$ be a homomorphism of minimal ttgs. Then the following statements are equivalent:

(a) $\phi$ is a PI extension;

(b) $\phi$ is a $\mathrm{C}^{\prime}$-extension;

(c) $\phi$ is an $n-\mathrm{C}^{\prime}$-extension for some $n \in \mathrm{N}, n \geq 2$.

(d) $\phi$ is an $n$-C'-extension for every $n \in \mathbf{N}, n \geq 2$.

The proof of 3.1 requires several steps that will be formulated in separate lemmas. The straightforward proofs of the following remark will be omitted.

3.2. Remark: (a) A weakly mixing Bc extension that satisfies the $\mathrm{C}^{\prime}$ condition is an isomorphism.

(b) Let $\phi$ and $\psi$ be extensions of minimal ttgs such that $\psi \circ \phi$ is an $n$ - $\mathrm{C}^{\prime}$-extension. Then $\phi$ is an $n$ - $\mathrm{C}^{\prime}$-extension.

(c) Let $\left\{\phi_{\alpha}: \mathscr{X}_{\alpha} \rightarrow \mathscr{Y} \mid \alpha<\nu\right\}$ be an inverse system of $n$-C'-extensions. Then $\phi:=$ inv $\lim \phi_{\alpha}$ is an $n$ - $\mathrm{C}^{\prime}$-extension.

3.3. Lemma. Let $\phi$ be an $n$ - $\mathrm{C}^{\prime}$-extension for some $n \geq 2$. Then $\phi$ is an $m-\mathrm{C}^{\prime}$-extension for every $m \in \mathbf{N}$ with $2 \leq m \leq n$.

Proof. Suppose for $n \geq 3, \phi$ is an $n$ - $\mathrm{C}^{\prime}$-extension. We shall prove that $\phi$ is an $n-1-\mathrm{C}^{\prime}$-extension.

Let $A=\overline{T A}$ be an ergodic subset of $R_{\phi}^{n-1}$ with a dense set of almost periodic points. Let $\pi: R_{\phi}^{n} \rightarrow R_{\phi}^{n-1}$ be the projection. By the proof of 2.5 there exists an $A^{\prime}=\overline{T A^{\prime}} \subseteq R_{\phi}^{n}$ which is ergodic such that $\left.\pi\right|_{A^{\prime}}: A^{\prime} \rightarrow A$ is a semi-open surjection and, moreover, such that $A^{\prime}$ does not contain a proper closed invariant subset that is mapped onto $A$. Let $U \subseteq A^{\prime}$ be open. Suppose that $U$ contains no almost periodic points. Then $T U$ contains no almost periodic points, which means that $A^{\prime} \backslash T U$ contains all 
almost periodic points that are in the preimages of the almost periodic points in $A$. This shows that $A^{\prime} \backslash T U$ is a closed invariant subset which is mapped onto $A$. Hence $A^{\prime}=A^{\prime} \backslash T U$, so $U=\varnothing$. Consequently, $A^{\prime}$ has a dense set of almost periodic points. As $\phi$ is an $n$ - $\mathrm{C}^{\prime}$-extension, it follows that $A^{\prime}$ is minimal, so $A$ is minimal.

Using the same reasoning as in the proof of 3.3 one shows:

3.4. LemMa. A factor of an $n-\mathrm{C}^{\prime}$-extension is an $n-\mathrm{C}^{\prime}$-extension; i.e.: If $\psi \circ \phi$ is an $n-\mathrm{C}^{\prime}$-extension then $\psi$ is an $n-\mathrm{C}^{\prime}$-extension too.

3.5. LeMmA. Let $\phi: \mathscr{X} \rightarrow \mathscr{Y}$ and $\psi: \mathscr{Y} \rightarrow \mathscr{Z}$ be homomorphisms of minimal ttgs and let $n \in \mathbf{N}$ with $n \geq 2$.

(a) If $\psi$ is an $n$ - $\mathrm{C}^{\prime}$-extension and if $\phi$ is almost periodic then $\psi \circ \phi$ is an $n-C^{\prime}$-extension.

(b) If $\psi$ is an $n$ - $\mathrm{C}^{\prime}$-extension and if $\phi$ is proximal then $\psi \circ \phi$ is an $n-C^{\prime}$-extension.

Proof. (a) Let $A \subseteq R_{\psi \circ \phi}^{n}$ be ergodic with a dense set of almost periodic points. Then $\phi^{n}[A] \subseteq R_{\psi}^{n}$ is ergodic with a dense set of almost periodic points. As $\psi$ is an $n$ - $\mathrm{C}^{\prime}$-extension, $\phi^{n}[A]$ is minimal. As $\phi^{n}$ : $A \rightarrow \phi^{n}[A]$ is an almost periodic extension of a minimal set and as $A$ is ergodic, it follows from 2.1 that $A$ is minimal.

(b) Suppose $\psi$ is an $n$ - $\mathrm{C}^{\prime}$-extension and let $A=\overline{T A} \subseteq R_{\psi \circ \phi}^{n}$ be ergodic with a dense set of almost periodic points. Then $\phi^{n}[A] \subseteq R_{\psi}^{n}$ is ergodic with a dense set of almost periodic points. As $\psi$ was $n-\mathrm{C}^{\prime}, \phi^{n}[A]$ is minimal. By proximality of the map $\phi^{n}, A$ has a unique minimal subset; hence $A$ is minimal.

By now we are able to prove Theorem 3.1:

Proof (of 3.1). The implication (d) $\Rightarrow$ (c) is trivial and (c) $\Rightarrow(b)$ is just 3.3.

(b) $\Rightarrow$ (a). Suppose $\phi$ is a $C^{\prime}$-extension and consider the canonical PI shadow diagram for $\phi$ ([EGS 75], [V 77]):

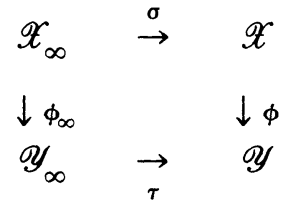

where $\sigma$ is proximal, $\tau$ is strictly PI and $\phi_{\infty}$ is a weakly mixing Bc extension ([V 77] 2.1.3). 
By 3.5(b), $\phi \circ \sigma$ is a $C^{\prime}$-extension; and, by 3.2(b), $\phi_{\infty}$ turns out to be a $\mathrm{C}^{\prime}$-extension. Hence, by 3.2(a), $\phi_{\infty}$ is an isomorphism; which shows that $\phi$ is a PI extension.

(a) $\Rightarrow$ (d). Let $\phi$ be a PI extension and let $\theta: \mathscr{W} \rightarrow \mathscr{Y}$ be a strictly PI-extension such that $\theta=\phi \circ \kappa$ for some $\kappa: \mathscr{W} \rightarrow \mathscr{X}$. Let $n \in \mathbf{N}, n \geq 2$. Then, by 3.5(a) and (b) and by 3.2(c), it follows that $\theta$ is an $n$ - $\mathrm{C}^{\prime}$-extension. Hence, by $3.4, \phi$ is an $n-\mathrm{C}^{\prime}$-extension. As $n$ was chosen arbitrarily, this proves the theorem.

One might wonder how this characterization for PI extensions relates to other known ones. The relation to the PI characterization in [EGS 75] is obvious and it is made through [ $\mathbf{V}$ 77] 2.6.3. In order to relate our characterization to the one in [MN 80] we need the following definition.

Let $\phi: \mathscr{X} \rightarrow \mathscr{Y}$ be a homomorphism of minimal ttgs, let $y \in Y$ and let $\left\{t_{i}\right\}_{i}$ be a net in $T$. For a closed subset $K \subseteq \phi^{\leftarrow}(y)$ and for $x$ and $z$ elements of $K$, we say that $x$ is strongly regionally proximal to $y$ in $K$ with respect to the net $\left\{t_{i}\right\}_{i}$ if there are $z_{i} \in K$ such that $t_{i} x \rightarrow x, z_{i} \rightarrow z$ and $t_{i} z_{i} \rightarrow x$. In other words: for $x$ and $z$ we can find a regionally proximal making net in $K \times K$ such that the coordinate tending to $x$ is fixed $(x)$ and such that the net in $T$ chasing it to the diagonal is the prescribed one. Notation: $x \in \operatorname{SRP}\left(\phi, K, z,\left\{t_{i}\right\}_{i}\right)$.

This definition is slightly different from the one in [MN 80]. First, because it is a generalization to the relativized case. (Note that the results in the first section of [MN 80] can easily be transferred to the relativized case.) Second, because it takes a net into account, which is necessary to state the characterization in [MN 80] as strong as it was proven.

3.6. THEOREM ([MN 80] 1.1). Let $\phi: \mathscr{X} \rightarrow \mathscr{Y}$ be a homomorphism of minimal ttgs. Then $\phi$ is not a PI extension iff for some $w \in J$ there is a nontrivial set $K=\overline{w K} \subseteq \phi^{\leftarrow}(y)$, for some $y=w y$, such that for some (every) net $\left\{t_{i}\right\}_{i} \rightarrow w$ in $T$ and for every subnet $\left\{s_{i}\right\}_{i}$ of $\left\{t_{i}\right\}_{i}$ there is $a$ subnet $\left\{t_{i}^{*}\right\}_{i}$ of $\left\{s_{i}\right\}_{i}$ such that $x \in \operatorname{SRP}\left(\phi, K, z,\left\{t_{i}^{*}\right\}_{i}\right)$ for every $x \in w K$ and $z \in K$. (Nontriviality for $K$ means $|K| \geq 2$.)

We shall show that such a $K$ (as in the theorem) defines an ergodic subset $\overline{T(K \times K)}$ in $R_{\phi}$ with a dense set of almost periodic points. It then follows that if $\phi$ is PI, $T(K \times K)$ has to be minimal, hence $K$ has to be trivial. This illustrates how our characterization relates to the one in [MN 80]. 
First note that $w K \times w K$ is dense in $K \times K$, so $T(w K \times w K)$ is a dense set of almost periodic points in $\overline{T(K \times K)} \subseteq R_{\phi}$. Let $U$ $\subseteq \overline{T(K \times K)}$ be open, then for some $t_{1} \in T, t_{1} U \cap K \times K \neq \varnothing$ and open in $K \times K$. Then there is a point $\left(x_{1}, x_{2}\right)=\left(w x_{1}, w x_{2}\right) \in K \times K \cap$ $t_{1} U$. Let $V \subseteq \overline{T(K \times K)}$ be open, then for some $t_{2} \in T, t_{2} V \cap K \times K \neq$ $\varnothing$. Choose $\left(z_{1}, z_{2}\right) \in t_{2} V \cap K \times K$. Let $\left\{s_{i}\right\}_{i}$ be a subnet of $\left\{t_{i}\right\}_{i} \rightarrow w$ such that for certain $z_{1}^{l} \in K$ we have $z_{1}^{l} \rightarrow z_{1}, s_{i} z_{1}^{i} \rightarrow x_{1}$. Let $\left\{t_{i}^{*}\right\}_{i}$ be a subnet of $\left\{s_{i}\right\}_{i} \rightarrow w$ such that for certain $z_{2}^{i} \in K$ we have $z_{2}^{i} \rightarrow z_{2}$ and $t_{l}^{*} z_{2}^{i} \rightarrow x_{2}$. Then $\left(z_{1}^{i}, z_{2}^{i}\right) \rightarrow\left(z_{1}, z_{2}\right)$ and $t_{i}^{*}\left(z_{1}^{i}, z_{2}^{i}\right) \rightarrow\left(x_{1}, x_{2}\right)$. So for certain $i_{0}, t_{i_{0}}^{*}\left(z_{1}^{i_{0}}, z_{2}^{i_{0}}\right) \in t_{1} U$ and $\left(z_{1}^{i_{0}}, z_{2}^{i_{0}}\right) \in t_{2} V$. Hence $t_{i_{0}}^{*} t_{2} V \cap t_{1} U \neq \varnothing$, which shows ergodicity of $\overline{T(K \times K)}$.

Note that for non-PI extensions it is easy to find a nontrivial $K=\overline{w K}$ as in 3.6 , namely $K=\overline{w F_{\infty} x_{0}}$ where $F=\mathbb{B S}\left(\mathscr{Y}, \phi\left(x_{0}\right)\right)$ and $w \in J$ such that $\left\langle\overline{w F_{\infty}}, F_{\infty}\right\rangle$ is a minimal right $\operatorname{ttg}$ for $F_{\infty}$.

IV. A characterization of HPI extensions. Although in the metric case HPI extensions are known as the pointdistal extensions, there does not exist a satisfying intrinsic characterization for HPI extensions of nonmetric minimal ttgs. (The most interesting up to now is the one in [AG 77] Thm. III.2).

Note that universal PI extensions may be characterized in terms of quasifactors of $\mathscr{M}$. The known characterization of the universal HPI extensions in terms of quasifactors of $\mathscr{M}$, however, is rather artificial ([Wo 82] V.4).

In this section we shall give a characterization in the same spirit as the PI characterization in 3.1. The next definition is the HPI counterpart of the one for $n-\mathrm{C}^{\prime}$-extensions in the previous section: Let $n \in \mathbf{N}, n \geq 2$ and let $\phi: \mathscr{X} \rightarrow \mathscr{Y}$ be a homomorphism of minimal ttgs. Then $\phi$ is said to satisfy the $n$-ESOM property iff every ergodic sub $\operatorname{ttg} A=\overline{T A} \subseteq R_{\phi}^{n}$ such that every projection $\pi_{i}: \mathscr{A} \rightarrow \mathscr{X}$ is semi-open is minimal. If $\phi$ is a 2-ESOM extension, we say $\phi$ is an ESOM extension.

4.1. REMARK. Let $\phi: \mathscr{X} \rightarrow \mathscr{Y}$ be a homomorphism of minimal ttgs.

(a) If $\phi$ is an ESOM extension, then $\phi$ is a PI extension.

(b) If $\phi$ is a pointdistal PI extension then $\phi$ is an ESOM extension.

Proof. (a) Let $A \subseteq R_{\phi}$ be ergodic with a dense set of almost periodic points. Then, by $2.4(\mathrm{~b})$, we know that the projections $\pi_{i}: A \rightarrow \mathscr{X}$ are semi-open. So by the definition of ESOM, $A$ is minimal. Hence, by $3.1, \phi$ is a PI extension. 
(b) Let $A=\overline{T A} \subseteq R_{\phi}$ be ergodic with semi-open projections to $\mathscr{X}$. Let $U \subseteq A$ be open and let $V=\left(\pi_{1}[U]\right)^{\circ} \subseteq X$ then $V$ contains a $\phi$-distal point $x$. So there is a point $(x, z) \in U$ and as $x$ is $\phi$-distal, $(x, z)$ is an almost periodic point. This shows that $A$ has a dense set of almost periodic points. As $\phi$ is PI, it follows from 3.1 that $A$ is minimal. Hence $\phi$ is an ESOM map.

After our main result (4.8) it follows that a pointdistal PI extension is $n$-ESOM for every $n \in \mathbf{N}, n \geq 2$ (which, by then, is obvious).

The straightforward proofs of the following remark are omitted.

4.2. REMARK. (a) A weakly mixing ESOM extension $\phi$ of minimal ttgs which is either an open- or a BC extension is an isomorphism (use 2.4(b), (c)).

(b) Let $\phi$ and $\psi$ be extensions of minimal ttgs such that $\psi \circ \phi$ is an $n$-ESOM-extension. Then $\phi$ is an $n$-ESOM-extension.

(c) Let $\left\{\phi_{\alpha}: \mathscr{X}_{\alpha} \rightarrow \mathscr{Y} \mid \alpha<\nu\right\}$ be an inverse system of $n$-ESOM-extensions. Then $\phi=\operatorname{inv} \lim \phi_{\alpha}$ is an $n$-ESOM-extension.

4.3. Lemma. Let $\phi: \mathscr{X} \rightarrow \mathscr{Y}$ and $\psi: \mathscr{Y} \rightarrow \mathscr{Z}$ be homomorphisms of minimal ttgs and let $n \in \mathbf{N}$ with $n \geq 2$.

(a) If $\psi$ is an $n$-ESOM-extension and $\phi$ is almost periodic, then $\psi \circ \phi$ is an $n$-ESOM-extension.

(b) If $\psi$ is an $n$-ESOM-extension and $\phi$ is highly proximal, then $\psi \circ \phi$ is an $n$-ESOM-extension.

Proof. (a) Let $A=\overline{T A}$ be an ergodic subset of $R_{\psi \circ \phi}^{n}$ with semi-open projections to $\mathscr{X}$. Then $\phi^{n}[A]$ is an ergodic subset of $R_{\psi}^{n}$. That the projections to $\mathscr{Y}$ are semi-open follows from the fact that $\phi: \mathscr{X} \rightarrow \mathscr{Y}$ as a homomorphism of minimal ttgs is semi-open (2.4(b)). As $\psi$ is $n$-ESOM, $\phi^{n}[A]$ is minimal. Hence, by $2.1, A$ is minimal; so $\psi \circ \phi$ is an $n$-ESOM-extension.

(b) Let $A=\overline{T A}$ be an ergodic subset of $R_{\psi \circ \phi}^{n}$ with semi-open projections to $\mathscr{X}$. Then $\phi^{n}[A]$ is ergodic with semi-open projections to $\mathscr{Y}$. Hence, as $\psi$ is $n$-ESOM, $\phi^{n}[A]$ is minimal in $R_{\psi}^{n}$.

Let $U_{i} \subseteq X$ be open with $U_{1} \times \cdots U_{n} \cap A \neq \varnothing$. By semi-openness of $\pi_{1}$, there is a nonempty open $V_{1} \subseteq\left(\pi_{1}\left[U_{1} \times \cdots \times U_{n} \cap A\right]\right)^{\circ}$. As $\phi$ is irreducible, there is a nonempty open $W_{1}=\phi^{\leftarrow} \phi\left[W_{1}\right] \subseteq V_{1}$ and $\phi\left[W_{1}\right]$ is nonempty and open in $Y$. Note that $W_{1} \times U_{2} \times \cdots \times U_{n} \cap A \neq \varnothing$ and open. By semi-openness of $\pi_{2}$ and irreducibility of $\phi$ there is an open $W_{2}$ 
in $X$ with $W_{2}=\phi^{\leftarrow} \phi\left[W_{2}\right] \subseteq U_{2}$ and $W_{1} \times W_{2} \times U_{3} \times \cdots \times U_{n} \cap A \neq$ $\varnothing$ and open. Proceeding this way, there are open $W_{i} \subseteq U_{i}$ with $W_{i}=$ $\phi^{\leftarrow} \phi\left[W_{i}\right]$ and $W_{1} \times \cdots \times W_{n} \cap A \neq \varnothing$. From the choice of the $W_{i}$ it follows that $W_{1} \times \cdots \times W_{n} \cap A$ contains a full preimage of some $b \in$ $\phi^{n}[A]$ under the map $\left.\phi^{n}\right|_{A}: A \rightarrow \phi^{n}[A]$.

As this is true for every open $U=U_{1} \times \cdots \times U_{n} \cap A$ in $A$, it follows that $\left.\phi^{n}\right|_{A}: A \rightarrow \phi^{n}[A]$ is irreducible. So, by $2.3, A$ is minimal; which proves that $\psi \circ \phi$ is an $n$-ESOM extension.

In order to show that every HPI extension satisfies the $n$-ESOM property we just have to prove that a factor of an $n$-ESOM-extension under a highly proximal map is an $n$-ESOM-extension.

4.4. LemmA. Let $\phi: \mathscr{X} \rightarrow \mathscr{Y}$ and $\psi: \mathscr{Y} \rightarrow \mathscr{Z}$ be homomorphisms of minimal ttgs with $\phi$ highly proximal. If $\psi \circ \phi$ is $n$-ESOM then $\psi$ is an $n$-ESOM extension.

Proof. Let $A=\overline{T A} \subseteq R_{\psi}^{n}$ be ergodic with semi-open projections to $\mathscr{Y}$. By 2.5 , there is an ergodic $A^{\prime}=T A^{\prime} \subseteq R_{\psi \circ \phi}^{n}$ such that $\left.\phi^{n}\right|_{A^{\prime}}: A^{\prime} \rightarrow A$ is a semi-open surjection. We shall show that the projections from $A^{\prime}$ onto $\mathscr{X}$ are semi-open. It then follows from the $n$-ESOM property for $\psi \circ \phi$ that $A^{\prime}$ is minimal; hence $A$ as a factor of $A^{\prime}$ is minimal. So $\psi$ turns out to satisfy the $n$-ESOM property.

Let $U \subseteq A^{\prime}$ be open and choose an open $\varnothing \neq V \subseteq \bar{V} \subseteq U$. By semi-openness of $\left.\phi^{n}\right|_{A^{\prime}}, \phi^{n}[V]$ has a nonempty interior in $A$. So, by semi-openness of the $i$ th projection $\pi_{l}^{Y}: A \rightarrow \mathscr{Y}$, it follows that $\left(\pi_{i}^{Y} \circ \phi^{n}[V]\right)^{\circ}=W$ is nonempty in $Y$. But then $\pi_{i}^{Y} \circ \phi^{n}[\bar{V}]=\phi \circ \pi_{i}^{X}[\bar{V}]$ has a nonempty interior in $Y$; and so for some finite $F \subseteq T$ we have

$$
Y=F \cdot\left(\phi \circ \pi_{i}^{X}[\bar{V}]\right)=\phi \circ \pi_{i}^{X}[F \bar{V}] .
$$

(Here $\pi_{i}^{X}$ is the $i$ th projection from $A^{\prime}$ onto $\mathscr{X}$ ). As $\phi$ is irreducible, $\pi_{i}^{X}[F \bar{V}]=X=F \cdot \pi_{i}^{X}[\bar{V}]$. But then $\pi_{i}^{X}[\bar{V}]$ has a nonempty interior $(F$ is finite); and so $\pi_{i}^{X}[U]$ has a nonempty interior in $X$. As this holds for every $i \leq n$, it follows that all projections from $A^{\prime}$ onto $\mathscr{X}$ are semi-open.

4.5. TheOREM. If $\phi: \mathscr{X} \rightarrow \mathscr{Y}$ is an HPI extension of minimal ttgs, then $\phi$ is an $n$-ESOM-extension for every $n \in \mathbf{N}, n \geq 2$.

Proof. Let $\theta: \mathscr{W} \rightarrow \mathscr{Y}$ be a strictly HPI extension and let $\xi: \mathscr{W} \rightarrow \mathscr{X}$ be highly proximal. Then, by 4.3 (a) and (b) and 4.2(c), $\theta$ is an $n$-ESOM-extension for every $n \in \mathbf{N}, n \geq 2$. As $\xi$ is highly proximal, the theorem follows from 4.4 . 
For the following (the converse of 4.5) we need some details about the EGS $(\phi)$ diagram for $\phi: \mathscr{X} \rightarrow \mathscr{Y}$ (see [EGS 75], [G 76], [V 77] and, mainly for reasons of notation and for useful details, [Wo 82]).

Let $\phi: \mathscr{X} \rightarrow \mathscr{Y}$ be a homomorphism of minimal ttgs. Let $M$ be a fixed minimal left ideal in the universal ambit $S_{T}$ considered as the universal minimal ttg $\mathscr{M}$ for $T$, and let $J$ denote the collection of idempotents in $M$.

Define the shadow diagram $\operatorname{EGS}(\phi)$ for $\phi$ as follows:

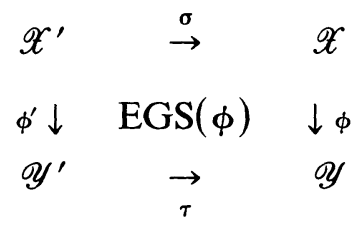

$Y^{\prime}=\left\{v \circ u \phi^{\leftarrow}(y) \mid y \in Y, v \in J_{y}\right\}$, where $J_{y}=\{w \in J \mid w y=y\}$ and $u \in$ $J$. (The circle operation refers to the action of $M$ on the hyperspace $2^{X}$ of $X$.) The map $\tau$ is defined by $\tau\left(v \circ u \phi^{\leftarrow}(y)\right)=y$. Then define $X^{\prime}=$ $\left\{\left(x, y^{\prime}\right) \mid x \in y^{\prime} \in Y^{\prime}\right\} \subseteq X \times Y^{\prime}$, and let $\tau$ and $\phi^{\prime}$ be the projections. Then $\mathscr{X}^{\prime}$ and $\mathscr{Y}^{\prime}$ are minimal ttgs, $\sigma$ and $\tau$ are proximal extensions and $\phi^{\prime}$ is a RIC extension.

Note that there are several equivalent descriptions for the elements of $Y^{\prime}: Y^{\prime}=\left\{v \circ v \phi^{\leftarrow}(y) \mid v \in J, y \in Y\right\} ; Y^{\prime}=\left\{p \circ u \phi^{\leftarrow} \phi\left(x_{0}\right) \mid p \in M\right\}$, here $x_{0} \in X$ is fixed and $u \in J ; Y^{\prime}=\left\{p \circ F x_{0} \mid p \in M\right\}$, where (again) $x_{0} \in X$ is fixed and $F=\mathscr{G}\left(\mathscr{Y}, \phi\left(x_{0}\right)\right)$ is the Ellis group of $\mathscr{Y}$ with respect to $\phi\left(x_{0}\right)$.

If $\phi: \mathscr{X}^{*} \rightarrow \mathscr{Y}^{*}$, then $\sigma$ and $\tau$ are open and with respect to the open sets in $X^{\prime}$ we have the following useful remark (in this situation):

As $X^{\prime} \subseteq X \times Y^{\prime}$ and as the projections are open $\left(\mathscr{X}=\mathscr{X}^{*} !\right), X^{\prime}$ has a base of open sets of the form $U \times V \cap X^{\prime} \neq \varnothing$, where $U$ and $V$ are open in $X$ and $Y^{\prime}$ and such that for every $u \in U$ there is a $v \in V$ with $(u, v) \in X^{\prime}$. In addition, note that for such basic open sets we have $\sigma\left[U \times V \cap X^{\prime}\right]=U$.

4.6. LEMMA. Let $\phi: \mathscr{X}^{*} \rightarrow \mathscr{Y}^{*}$ be a homomorphism of minimal ttgs and consider $\operatorname{EGS}(\phi)$. If $A \subseteq R_{\tau \circ \phi^{\prime}}$ is such that $\phi^{\prime} \times\left.\phi^{\prime}\right|_{A}: A \rightarrow R_{\tau}$ is a semi-open surjection, then $\sigma \times \sigma[A]$ has semi-open projections onto $\mathscr{X}^{*}$.

Proof. As $\tau$ is open, the projections $\pi_{i}^{Y^{\prime}}: R_{\tau} \rightarrow Y^{\prime}$ are semi-open, so the maps $\pi_{i}^{Y^{\prime}} \circ \phi^{\prime} \times \phi^{\prime}: A \rightarrow Y^{\prime}$ are semi-open.

Let $O \subseteq \sigma \times \sigma[A]$ be a nonempty open set in $\sigma \times \sigma[A]$, and let $W:=(\sigma \times \sigma)^{\leftarrow}[O] \cap A$ which is open in $A$. Choose basic open sets $W_{1}$ and $W_{2}$ in $X^{\prime}$ with $\varnothing \neq W_{1} \times W_{2} \cap A \subseteq W$. By semi-openness of 
$\pi_{1}^{Y^{\prime}} \circ \phi^{\prime} \times \phi^{\prime}: A \rightarrow Y^{\prime}$, we may find an open $\tilde{W}_{1} \subseteq W_{1}$ such that for every $w_{1} \in \tilde{W}_{1}$ there is a $w_{2} \in W_{2}$ with $\left(w_{1}, w_{2}\right) \in A$. Without loss of generality $\tilde{W}_{1}$ may be chosen to be basic open in $X^{\prime}$. Say $\tilde{W}_{1}=$ $U_{1} \times V_{1} \cap X^{\prime}$ and $W_{2}=U_{2} \times V_{2} \cap X^{\prime}$. We show that $U_{1} \subseteq \pi_{1}^{X}[O]$; hence it follows that $\pi_{1}^{X}: \sigma \times \sigma[A] \rightarrow \mathscr{X}^{*}$ is semi-open:

Let $u \in U_{1}$; then, by the discussion above, there is a $v \in V$ with $w=(u, v) \in X^{\prime}$. As $w \in \tilde{W}_{1}$, there is a $w_{2} \in W_{2}$ with $\left(w, w_{2}\right) \in W \subseteq A$, say $w_{2}=\left(u_{2}, v_{2}\right)$. Clearly $\sigma \times \sigma\left(w, w_{2}\right)=\left(u, u_{2}\right) \in \sigma \times \sigma[W] \subseteq O$, which shows that $U_{1} \subseteq \pi_{1}^{X}[O]$, hence that $\pi_{1}^{X}$ is semi-open.

Similarly, using semi-openness of $\pi_{2}^{Y^{\prime}} \circ \phi^{\prime} \times \phi^{\prime}: A \rightarrow Y^{\prime}$, one shows that $\pi_{2}^{X}$ is semi-open.

4.7. LeMMA. Let $\phi: \mathscr{X}^{*} \rightarrow \mathscr{Y}^{*}$ be an ESOM extension, then $\phi$ is a RIC extension.

Proof. Consider the shadow diagram $\operatorname{EGS}(\phi)$ for $\phi$.

$\begin{array}{ccc}\mathscr{X}^{\prime} & \stackrel{\sigma}{\rightarrow} & \mathscr{X}^{*} \\ \phi^{\prime} \downarrow & \searrow \psi & \downarrow \phi \\ \mathscr{Y}^{\prime} & \rightarrow & \mathscr{Y}^{*}\end{array}$

As $\phi \circ \sigma=\psi=\tau \circ \phi^{\prime}$ is open and $\tau$ is proximal, $R_{\psi \tau}$ is ergodic ([Wo 82] VII.2.14). By 2.5, there is an ergodic subset $A \subseteq R_{\psi}$ such that $1 \times \phi^{\prime}$ : $A \rightarrow R_{\psi \tau}$ is a semi-open surjection. As $\phi^{\prime} \times 1: R_{\psi \tau} \rightarrow R_{\tau}$ is an open map, $\phi^{\prime} \times \phi^{\prime}: A \rightarrow R_{\tau}$ is semi-open. So, by 4.6, $\sigma \times \sigma[A]$ has semi-open projections onto $\mathscr{X}^{*}$. Since $\sigma \times \sigma[A]$ is ergodic and $\phi$ is an ESOM extension, it follows that $\sigma \times \sigma[A]$ is minimal, say $\sigma \times \sigma[A]=\overline{T\left(x_{0}, \alpha x_{0}\right)}$ for some $x_{0} \in u \mathscr{X}^{*}$ and $\alpha \in F$. Here $F=\mathscr{S}\left(\mathscr{Y}^{*}, \phi\left(x_{0}\right)\right)$ is the Ellis group of $\mathscr{Y}^{*}$ with respect to $\phi\left(x_{0}\right)$. Let $H=\mathscr{S}\left(\mathscr{X}^{*}, x_{0}\right)$ and let $K=\bigcap\left\{f H f^{-1} \mid f \in F\right\}$ be the largest normal subgroup of $F$ which is contained in $H$.

We shall now construct an ergodic subset $\tilde{A}$ of $R_{\psi}$ such that $1 \times \phi^{\prime}$ : $\tilde{A} \rightarrow R_{\psi \tau}$ again is semi-open and, what is even more important, such that $\Delta_{X} \subseteq \sigma \times \sigma[\tilde{A}]$. As, by 4.6 , the projections of $\sigma \times \sigma[\tilde{A}]$ are semi-open, it follows by the ESOM property for $\phi$ that $\Delta_{X}=\sigma \times \sigma[\tilde{A}]$.

Consider $\mathfrak{A}(K)$, the universal proximal extension of ttgs with Ellis group $K$ and define $\kappa: \mathfrak{U}(K) \rightarrow X^{\prime}$ by $\kappa(p \circ K)=\left(p x_{0}, p \circ u \phi \leftarrow \phi\left(x_{0}\right)\right)$. Then, by 2.5, there is an ergodic subset $B$ of $R_{\psi \circ \kappa}$ such that $\kappa \times \kappa: B \rightarrow A$ is a semi-open surjection. Define the endomorphism $\rho_{\alpha^{-1}}: \mathfrak{A}(K) \rightarrow \mathfrak{A}(K)$ by $p \circ K \mapsto p \alpha^{-1} \circ K$. As $K \nless F$ and $\alpha^{-1} \in F$ it follows that $\rho_{\alpha^{-1}}$ is a well defined isomorphism. Then define $\tilde{B} \subseteq R_{\psi \circ \kappa}$ by $\tilde{B}=1 \times \rho_{\alpha^{-1}}[B]$. As 
$\tilde{B}$ and $B$ are isomorphic, $\tilde{B}$ is ergodic. Let $\tilde{A} \subseteq R_{\psi}$ be defined as $\tilde{A}=\kappa \times \kappa[\tilde{B}]$. Then $\tilde{A}$ is ergodic. As $\alpha \in F, p \alpha^{-1} \circ u \phi^{\leftarrow} \phi\left(x_{0}\right)=$ $p \circ u \alpha^{-1} \phi \leftarrow \phi\left(x_{0}\right)=p \circ u \phi^{\leftarrow} \phi\left(x_{0}\right)$, so the difference between $A$ and $\tilde{A}$ is only in the "third" coordinate which is filtered out after the "second" projection $\phi^{\prime}$. Hence $1 \times \phi^{\prime}[\tilde{A}]=R_{\psi \tau}$.

Let $O \subseteq \tilde{A}$ be relatively open. Then by continuity of $\kappa \times\left.\kappa\right|_{B}$, by isomorphy of $1 \times \rho_{\alpha^{-1}}$ and by semi-openness of $\kappa \times\left.\kappa\right|_{B}$ it follows that there exists an open $O^{\prime} \subseteq A$ with $1 \times \phi^{\prime}\left[O^{\prime}\right] \subseteq 1 \times \phi^{\prime}[O]$. As $1 \times\left.\phi^{\prime}\right|_{A}$ is semi-open, also $1 \times\left.\phi^{\prime}\right|_{\tilde{A}}$ is semi-open. Then, by $4.6, \sigma \times \sigma[\tilde{A}]$ has semiopen projections, so $\sigma \times \sigma[\tilde{A}]$ is minimal. Since $\left(x_{0}, \alpha x_{0}\right) \in \sigma \times \sigma[A]$, $\left(x_{0}, x_{0}\right)=\left(x_{0}, \alpha^{-1} \alpha x_{0}\right) \in \sigma \times \sigma[\tilde{A}]$. So $\sigma \times \sigma[\tilde{A}]=\Delta_{X}$. Note that this implies that an element $a \in \tilde{A}$ has the form

$$
a=\left(\left(x, v \circ u \phi^{\leftarrow} \phi(x)\right),\left(x, w \circ u \phi^{\leftarrow} \phi(x)\right)\right) \quad \text { with } v, w \in J_{\phi(x)} .
$$

This enables us to show that $R_{\phi}=\overline{J R_{\phi}}$ and better, that $\phi$ is RIC.

Let $\left(x_{1}, x_{2}\right) \in R_{\phi}$ then there are $v, w \in J_{\phi\left(x_{1}\right)}$ such that $x_{1} \in$ $v \circ u \phi \leftarrow \phi\left(x_{1}\right)$ and $x_{2} \in w \circ u \phi^{\leftarrow} \phi\left(x_{1}\right)$. As

$$
z=\left(\left(x_{1}, v \circ u \phi^{\leftarrow} \phi\left(x_{1}\right)\right), w \circ u \phi^{\leftarrow} \phi\left(x_{1}\right)\right) \in R_{\psi \tau},
$$

there is an $a \in A$ with $1 \times \phi^{\prime}(a)=z$. But $\sigma \times \sigma(a)=\left(x_{1}, x_{1}\right)$ so $x_{1} \in$ $w \circ u \phi \leftarrow \phi\left(x_{1}\right)$ for

$$
a=\left(\left(x_{1}, v \circ u \phi^{\leftarrow} \phi\left(x_{1}\right)\right),\left(x_{1}, w \circ u \phi^{\leftarrow} \phi\left(x_{1}\right)\right)\right)
$$

and $\left(x_{1}, w \circ u \phi^{\leftarrow} \phi\left(x_{1}\right)\right) \in X^{\prime}$. Hence $x_{1}$ and $x_{2}$ are elements of $w \circ u \phi^{\leftarrow} \phi\left(x_{1}\right)$ and so $\left(x_{1}, x_{2}\right) \in \overline{J R_{\phi}}$.

Moreover, as $x_{1}$ and $x_{2}$ were chosen arbitrarily it follows that for every $x_{1} \in X^{*}, x_{1} \in \bigcap\left\{w \circ u \phi^{\leftarrow} \phi\left(x_{1}\right) \mid w \in J_{\phi\left(x_{1}\right)}\right\}$. But then, as is easily seen, $\phi^{\leftarrow} \phi\left(x_{1}\right)=w \circ u \phi^{\leftarrow} \phi\left(x_{1}\right)$ for every $w \in J_{\phi\left(x_{1}\right)}$ and for every $x_{1} \in$ $X^{*}$, which proves that $\phi$ is a RIC extension.

Note how the lemma above resembles the characterization of HPI extensions in ([AG 77]] Thm. III.2).

4.8. TheOREM. Let $\phi: \mathscr{X} \rightarrow \mathscr{Y}$ be a homomorphism of minimal ttgs. Then the following statements are equivalent:

(a) $\phi$ is an HPI extension;

(b) $\phi$ is an ESOM extension;

(c) $\phi$ is an $n$-ESOM extension for every $n \in \mathbf{N}, n \geq 2$. 
Proof. (a) $\Rightarrow$ (c). Is just Theorem 4.5.

(c) $\Rightarrow$ (b). Is trivial.

(b) $\Rightarrow$ (a). Consider $\phi^{*}$ and note that $\phi^{*}$ is an HPI extension iff $\phi$ is an HPI extension. Let $\psi$ be the maximal HPI factor under $\phi^{*}$, say $\phi^{*}=\theta \circ \psi$ and remark that $\theta=\theta^{*}, \psi=\psi^{*}$ and $\theta^{*}$ does not admit nontrivial almost periodic factors (maximality of $\psi^{*}$ ). By $4.2(\mathrm{~b}), \theta^{*}$ is an ESOM extension and, by $4.7, \theta^{*}$ is a RIC extension. So, by [V 77] 2.6.3, $\theta^{*}$ is weakly mixing, i.e., $R_{\theta^{*}}$ is ergodic. As $\theta^{*}$ is open, the projections from $R_{\theta^{*}}$ onto $\mathscr{X}^{*}$ are semi-open. Hence $R_{\theta^{*}}$ is minimal and $\theta^{*}$ is an isomorphism, which proves that $\phi^{*}$ is an HPI extension and so $\phi$ is an HPI extension.

\section{REFERENCES}

[AG 77] J. Auslander and S. Glasner, Distal and highly proximal extensions of minimal flows, Indiana Math. J., 26 (1977), 731-749.

[AMWW 83] J. Auslander, D. C. McMahon, J. C. S. P. van der Woude and T. S. Wu, Weak disjointness and the equicontinuous structure relation, Ergodic Theory Dynamical Systems, 4 (1984), 323-351.

[AW 81] J. Auslander and J. C. S. P. van der Woude, Maximally highly proximal generators of minimal flows, Ergod. Th. \& Dynam. Sys. 1 (1981), 389-412.

[B 77] I. U. Bronstein, A characteristic property of PD-extensions, Bull. Akad. Stiince RSS Moldoven, 3 (1977), 11-15.

[EGS 75] R. Ellis, S. Glasner and L. Shapiro, Proximal isometric (PI-) flows, Advances in Math., 17 (1975), 213-260.

[G 76] S. Glasner, Proximal Flows, Lecture Notes in Math. 517, Springer-Verlag, New York 1976.

[MN 80] D. C. McMahon and L. J. Nachman, An intrinsic characterization for PI-flows, Pacific J. Math., 89 (1980), 391-403.

[V 77] W. A. Veech, Topological dynamics, Bull. Amer. Math. Soc., 83 (1977), $775-830$.

[Wi 70] A. Wilanski, Topology for Analysis, Xerox College Publishing, Toronto 1970.

[Wo 82] J. C. S. P. van der Woude, Topological dynamix, dissertation, Vrije Universiteit Amsterdam 1982. CWI tract 22, Center for Math. and Computer Sc., Amsterdam 1985.

Received December 2, 1983.

CASE WESTERN RESERVE UNIVERSITY

Cleveland, OH 44106

Current address: Technische Hogeschool Eindhoven

Eindhoven, The Netherlands 



\section{PACIFIC JOURNAL OF MATHEMATICS EDITORS}

\author{
V. S. VARADARAJAN (Managing Editor) \\ University of California \\ Los Angeles, CA 90024 \\ Charles R. DePrima \\ California Institute of Technology \\ Pasadena, CA 91125 \\ R. FINN \\ Stanford University \\ Stanford, CA 94305
}

\author{
HeRmanN FlaschKa \\ University of Arizona \\ Tucson, AZ 857.21
}

RAMESH A. GANGOLli

University of Washington

Seattle, WA 98195

ROBION KIRBY

University of California

Berkeley, CA 94720
C. C. MOORE

University of California

Berkeley, CA 94720

H. SAMELSON

Stanford University

Stanford, CA 94305

HAROLD STARK

University of California, San Diego

La Jolla, CA 92093

\section{ASSOCIATE EDITORS}
R. ARENS
E. F. BECKENBACH
B. H. NeumanN
F. WOLF
K. YoSHIDA
(1906-1982)

\section{SUPPORTING INSTITUTIONS}

\begin{abstract}
UNIVERSITY OF ARIZONA
UNIVERSITY OF BRITISH COLUMBIA

CALIFORNIA INSTITUTE OF TECHNOLOGY

UNIVERSITY OF CALIFORNIA

MONTANA STATE UNIVERSITY

UNIVERSITY OF NEVADA, RENO

NEW MEXICO STATE UNIVERSITY

OREGON STATE UNIVERSITY
\end{abstract}

\author{
UNIVERSITY OF OREGON \\ UNIVERSITY OF SOUTHERN CALIFORNIA \\ STANFORD UNIVERSITY \\ UNIVERSITY OF HAWAII \\ UNIVERSITY OF TOKYO \\ UNIVERSITY OF UTAH \\ WASHINGTON STATE UNIVERSITY \\ UNIVERSITY OF WASHINGTON
}

The Supporting Institutions listed above contribute to the cost of publication of this Journal, but they are not owners or publishers and have no responsibility for its content or policies.

Mathematical papers intended for publication in the Pacific Journal of Mathematics should be in typed form or offset-reproduced (not dittoed), double spaced with large margins. Please do not use built up fractions in the text of the manuscript. However, you may use them in the displayed equations. Underline Greek letters in red, German in green, and script in blue. The first paragraph must be capable of being used separately as a synopsis of the entire paper. In particular it should contain no bibliographic references. Please propose a heading for the odd numbered pages of less than 35 characters. Manuscripts, in triplicate, may be sent to any one of the editors. Please classify according to the scheme of Math. Reviews, Index to Vol. 39. Supply name and address of author to whom proofs should be sent. All other communications should be addressed to the managing editor, or Elaine Barth, University of California, Los Angeles, California 90024.

There are page-charges associated with articles appearing in the Pacific Journal of Mathematics. These charges are expected to be paid by the author's University, Government Agency or Company. If the author or authors do not have access to such Institutional support these charges are waived. Single authors will receive 50 free reprints; joint authors will receive a total of 100 free reprints. Additional copies may be obtained at cost in multiples of 50 .

The Pacific Journal of Mathematics is issued monthly as of January 1966. Regular subscription rate: $\$ 190.00$ a year (5 Vols., 10 issues). Special rate: $\$ 66.00$ a year to individual members of supporting institutions.

Subscriptions, orders for numbers issued in the last three calendar years, and changes of address should be sent to Pacific Journal of Mathematics, P.O. Box 969, Carmel Valley, CA 93924, U.S.A. Old back numbers obtainable from Kraus Periodicals Co., Route 100, Millwood, NY 10546.

The Pacific Journal of Mathematics at P.O. Box 969, Carmel Valley, CA 93924 (ISSN 0030-8730) publishes 5 volumes per year. Application to mail at Second-class postage rates is pending at Carmel Valley, California, and additional mailing offices. Postmaster: Send address changes to Pacific Journal of Mathematics, P.O. Box 969, Carmel Valley, CA 93924.

PUBLISHED BY PACIFIC JOURNAL OF MATHEMATICS, A NON-PROFIT CORPORATION

Copyright $\odot 1985$ by Pacific Journal of Mathematics 


\section{Pacific Journal of Mathematics}

Vol. 120, No. $2 \quad$ October, 1985

Philip Marshall Anselone and Mike Treuden, Regular operator

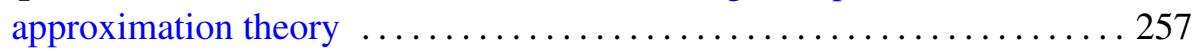

Giuseppe Baccella, Semiprime $\aleph-Q F 3$ rings $\ldots \ldots \ldots \ldots \ldots \ldots \ldots \ldots . \ldots \ldots$

Earl Robert Berkson and Thomas Alastair Gillespie, The generalized M.

Riesz theorem and transference $\ldots \ldots \ldots \ldots \ldots \ldots \ldots \ldots . \ldots 279$

Joachim Boidol, A Galois-correspondence for general locally compact

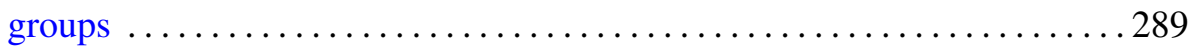

Joseph Eugene D'Atri, Josef Dorfmeister and Yan Da Zhao, The isotropy

representation for homogeneous Siegel domains ............... 295

C. Debiève, On Banach spaces having a Radon-Nikodým dual

Michael Aaron Freedman, Existence of strong solutions to singular

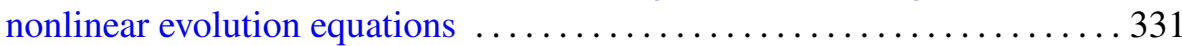

Francisco Jose Freniche, Grothendieck locally convex spaces of continuous

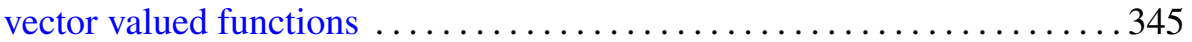

Hans-Peter Künzi and Peter Fletcher, Extension properties induced by complete quasi-uniformities . ............................ 357

Takaŝi Kusano, Charles Andrew Swanson and Hiroyuki Usami, Pairs of

positive solutions of quasilinear elliptic equations in exterior domains . . 385

Angel Rafael Larotonda and Ignacio Zalduendo, Spectral sets as Banach

manifolds

J. Martínez-Maurica and C. Pérez García, A new approach to the

Kreı̆n-Milman theorem

Christian Pommerenke, On the boundary continuity of conformal maps . . . 423

M. V. Subba Rao, Some Rogers-Ramanujan type partition theorems

Stephen Edwin Wilson, Bicontactual regular maps .........

Jaap C. S. P. van der Woude, Characterizations of (H)PI extensions

Kichoon Yang, Deformation of submanifolds of real projective space

Subhashis Nag, Errata: "On the holomorphy of maps from a complex to a

real manifold" 